\title{
Surgery for Good Prognosis Breast Cancers
}

\author{
Stuart A. McIntosh ${ }^{1}$ (D) \\ Accepted: 26 March 2021 / Published online: 21 April 2021 \\ (C) The Author(s) 2021
}

\begin{abstract}
Purpose of Review The introduction of mammographic screening programmes has resulted increasing numbers of women with small breast cancers with biologically favourable characteristics. Many of these cancers may represent overdiagnosis, with a resulting treatment burden for women and healthcare costs for providers. Here, current surgical approaches to the treatment of such tumours are reviewed, together with alternative approaches to their management.

Recent Findings The surgical treatment of small, screen-detected breast cancers with biologically favourable characteristics has been extrapolated from the management of symptomatic breast cancers. There is no prospective randomised evidence for conventional open surgery compared with other approaches in this setting. A number of minimally invasive techniques, most notable vacuum-assisted excision, have been described for the management of these tumours, but at present, there is a lack of high-quality evidence to support their routine use. There are currently ongoing randomised trials evaluating risk-adapted surgical and minimally invasive approaches to the management of good prognosis disease.

Summary It is possible that the surgical treatment of good prognosis screen-detected breast cancers may be de-escalated. However, high-quality evidence from ongoing prospective randomised trials will be required in order to change clinical practice.
\end{abstract}

Keywords Breast cancer $\cdot$ Breast screening $\cdot$ Surgery $\cdot$ Minimally invasive surgery $\cdot$ Vacuum-assisted excision

\section{Introduction}

Breast cancer is the most common female cancer worldwide, accounting for $11.6 \%$ of all malignancies, with an estimated 2.1 million cases in 2018 [1]. The incidence of breast cancer has continued to increase over the last few decades, with the most marked increases seen in areas with a historically low incidence of the disease [2]. However, in many countries where national breast screening programmes were introduced, there were short-term sharp increases in breast cancer incidence. For example, in England and Wales, a sharp increase in the age standardised incidence of breast cancer was seen in the population aged 50-64 following the introduction of screening for this age group; this increase was not mirrored

This article is part of the Topical Collection on Breast Cancer Imaging and Screening

Stuart A. McIntosh

s.mcintosh@qub.ac.uk

1 Patrick G Johnston Centre for Cancer Research, Queen's University Belfast, 97 Lisburn Road, Belfast BT9 7AE, UK in non-screened women [3]. Similar increases in incidence in women of screening age have been observed in the USA [4].

\section{Breast Screening and Overdiagnosis}

Since the inception of mammographic screening programmes, it is clear that there has been a disproportionate increase in the incidence of small breast cancers, without a comparable reduction in the number of larger tumours. Using US SEER data, Welch et al. demonstrated an increase in the incidence of tumours $<2 \mathrm{~cm}$ in size from 36 to $68 \%$, following the commencement of screening [5•]. Although a smaller proportion of larger tumours were seen after screening was introduced, this was as a direct consequence of diagnosis of more small tumours rather than due to a reduction in the numbers of large cancers being diagnosed.

Historically, tumour anatomical factors such as size and regional lymph node status have been used to guide decision-making about prognosis and adjuvant therapies; however, emerging data indicates that tumour biology rather than anatomy is key to breast cancer prognosis [6]. Indeed, Lannin and Wang demonstrated using SEER data that large, 
favourable breast cancers may in fact have a better prognosis than small tumours with unfavourable biological characteristics [7••]. Although conventional histopathological tumour features such as grade and receptor status may be considered rather crude reflections of tumour biology, similar relationships have been seen using molecular assays. For example, in the TAILORx trial, patients assessed as having a low recurrence score using the Oncotype Dx 21-gene assay had a similar excellent prognosis irrespective of tumour size $\leq 2 \mathrm{~cm}$ versus $>2 \mathrm{~cm}$ [8]. It is thus clear that a proportion of small tumours with favourable biological characteristics have an excellent prognosis, with 10-year breast cancer-specific survival approaching $100 \%$ in the large series reported by Lannin and Wang [7••]. There is therefore little doubt that a proportion of patients detected by mammographic screening represent overdiagnosis - that is, the diagnosis of a tumour which never becomes clinically apparent during the patient's lifetime.

An alternative definition proposed for overdiagnosis is that of Etzioni et al., who define overdiagnosis as that which occurs when the time to death from a cause other than cancer is less that the lead time - as the critical issue is not whether the tumour ultimately progresses, but rather whether it progresses within the lifetime of the patient [9]. Modelling by Lannin and Wang, using SEER data with different estimates of overdiagnosis, estimated lead times lying between 8.9 and 44.9 years for tumours with biologically favourable characteristics [7••]. Concerns around overdiagnosis and the consequent potential harms of breast screening led in the UK to the commissioning of an Independent Panel Review of screening [10]. Published in 2012, the Marmot review concluded that for 10,000 women screened at a three-yearly interval for 20 years, commencing at age 50, 43 breast cancer deaths would be prevented and 129 cases of non-invasive and invasive breast cancer would be overdiagnosed - so 1 breast cancer death would be prevented for about every 3 overdiagnosed cases of breast cancer.

Thus, it is very clear that mammographic screening programmes result in the identification of a sizeable group of patients with biologically favourable tumours, which are unlikely to progress in a patient's lifetime, but which will carry a significant psychological and treatment burden for these women.

\section{Surgical Treatment of Good Prognosis Breast Cancer}

Although in recent years there have been increasing attempts to personalise systemic therapies for breast cancer, there is currently no individualised approach to the surgical treatment of such patients. The surgical treatment of screen-detected breast cancers has been directly extrapolated from the management of symptomatic disease and has evolved from extensive surgical approaches such as radical mastectomy, through to the widespread introduction over the last 3 decades of breast conserving surgery, as introduced by Bernard Fisher and Umberto Veronesi in their pivotal trials $[11,12]$. The vast majority of patients with biologically favourable small breast cancers will be treated with breast conserving surgery - in the UK in 2018 , $89 \%$ of screen-detected invasive tumours less than $15 \mathrm{~mm}$ in maximum diameter underwent breast conserving procedures [13]. However, there remains low but not insignificant morbidity associated with breast conservation. Complications may include wound, infections, and thrombotic and bleeding complications, and in a large US series, the incidence of these was estimated at just under $2 \%$ [14]. Importantly, however, breast conservation surgery may be associated with poor cosmetic outcomes, which have been shown to have a concomitant impact on quality of life and psychosocial morbidity [15]. Furthermore, despite the fact that breast conserving surgery has been a standard part of the management of breast cancer for over two decades, until recently, there has been lack of consensus on what constitutes an adequate clear resection margin around the tumour, with up to 1 in 4 women reportedly undergoing a repeat procedure to attain wider margins [16]. Although consensus guidance has now been published on optimal margin width, there remains a significant proportion of women undergoing repeat surgery following breast conservation for good prognosis tumours [13, 17]. Such repeat surgery clearly carries the risks of additional discomfort, complications, and poorer aesthetic outcomes for these patients, as well as increased healthcare costs for providers.

Additionally, patients with screen-detected biologically favourable cancers generally undergo surgical staging of the axilla, in the form of an axillary sentinel lymph node biopsy. As with breast conserving surgery, the morbidity of SLNB is low but is not negligible, with a reported incidence of $5 \%$ for arm lymphoedema and $11 \%$ for sensory disturbance in an early randomised trial comparing the two [18]. However, the vast majority of patients with small, low-grade breast cancers do not have nodal disease, and consequently, such patients are unlikely to benefit from surgical axillary staging. Furthermore, it would appear that a low burden of axillary nodal disease can be controlled by adequate adjuvant therapies, as shown in the long-term follow-up from the American College of Surgeons Oncology Group Z0011 trial, where patients with a positive SLNB randomised to no further axillary surgery had no disadvantage in terms of axillary recurrence or overall survival at 10 years, when compared with those having completion ANC [19•]. Given that the majority of patients with nodal involvement can be identified following radiological and cytopathological assessment at diagnosis, there is increasing interest in potentially omitting surgically staging of the axilla in such cases. Retrospective data suggests that patients who do not undergo surgical axillary staging do not have high rates of regional recurrence and have favourable 
disease-free survival (1.9\% and $91.2 \%$ respectively at 10 years in one UK series) [20]. The omission of SLNB in early breast cancer is currently being investigated in several randomised controlled trials in Europe [21-23].

Therefore, there is clearly a population of patients diagnosed with early breast cancer, largely through mammographic screening programmes, which is likely to be overdiagnosed, and therefore, the use of standard surgical treatment for these cancers may well represent overtreatment, with attendant morbidity and healthcare costs. As highlighted by the UK Independent Panel on Breast Screening, there is a pressing clinical need for alternate treatment strategies for this patient group [10].

\section{Alternative Approaches to Treatment for Good Prognosis Tumours}

Given that the majority of the small, biologically favourable breast tumours detected in breast screening programmes may well represent overdiagnoses, there is no prospective evidence that open surgical treatment of these tumours is required. Thus, it is increasingly important that minimally invasive techniques be developed for use in this setting, in order to minimise the treatment burden for patients. Numerous image-guided ablation techniques, using energy to destroy tumour cells, including radiofrequency ablation, cryoablation, high-intensity focused ultrasound (HIFU), and laser ablation have been described in breast cancer and have been comprehensively reviewed in a recent meta-analysis [24•]. Although these techniques were shown to have high technical success rates $(96 \%)$, where the operator was able to complete the ablation procedure, technique efficacy (defined as complete ablation of the lesion of interest, following subsequent surgical excision of the lesion) was much lower in this meta-analysis, ranging from $49 \%$ for HIFU up to $90 \%$ for microwave ablation. However, these were all small studies (with a mean study size of 24 patients), and although mostly prospective, they were cohort studies with no control arm. Furthermore, ablation studies, by their nature, disrupt the tumour tissue, meaning that no tissue is available for histopathological assessment. An approach which excises rather than ablates the tumour may be more desirable, and the Intact Percutaneous Excision (IPEX) Registry Study describes a minimally invasive approach to the excision of small tumours, where radiofrequency basket capture is used to remove lesions intact for histopathological assessment, including margins [25•]. Using the Intact system, Whitworth et al. described a prospective cohort study of 72 small invasive breast cancers and 52 DCIS lesions excised using this methodology, achieving an $81 \%$ rate of clear resection margins following percutaneous excision [25•]. Again, however, this represents a prospective cohort study, and there is no doubt that in order to change clinical practice in this setting, prospective randomised evidence will be required.

\section{Vacuum-Assisted Excision of Small Breast Cancers}

Furthermore, many of the techniques discussed above for the minimally invasive treatment of small tumours require specialist equipment and expertise which is not readily available or indeed scalable across healthcare systems. However, vacuum-assisted needle biopsy and excision (VAB/VAE) is a technique which is in widespread use globally for the diagnosis and management of breast lesions. Vacuum-assisted excision uses a larger calibre needle than core needle biopsy (up to a $7 \mathrm{G}$ needle) and has the advantage that multiple cores of tissue can be taken with the needle remaining in situ after a single pass. It is generally carried out under local anaesthesia as an outpatient procedure and may be done under stereotactic or ultrasound guidance. Initially described in 1996 for diagnostic use, VAE has evolved and been utilised as a tool for the non-surgical excision of benign breast lesions [26-28]. However, the increased volumes of tissue obtainable using $\mathrm{VAB} / \mathrm{VAE}$ approaches has meant that the technique can be successfully used for the diagnosis and definitive management of lesions of uncertain malignant potential (BI-RADS3 lesions [29]). There is now a substantial weight of published data suggesting that VAE can be safely used for the management of such lesions, with complete excision possible in the majority of cases, enabling many women to avoid surgery [30, 31]. Fine et al. have suggested that post VAE imaging can accurately estimate complete lesion removal in over $90 \%$ of cases, and a recent report by Perretta et al. demonstrated a complete excision rate of BI-RADS3 lesions of $93.6 \%$ after VAE [32, 33]. Perretta et al. also noted that favourable US features such as circumscribed margins were associated with complete excision. Furthermore, data suggests that the approach is welltolerated and accepted by patients [34]. Indeed, in the UK, the use of VAB and VAE for the management of such lesions is now part of clinical guidance within the NHS Breast Screening Programme, and the technique is approved by the National Institute of Health and Care Excellence for the excision of benign lesions. [35].

It is clear therefore that there exists a group of small, screen-detected breast cancers with an indolent nature, many of which may represent overdiagnoses, and where there is a need to reduce the treatment burden for our patients. Vacuumassisted excision appears to be a widely available technique, with which there is extensive radiologist and surgeon experience and is acceptable to patients, which could potentially be developed for the treatment of such tumours. There is currently little data on the use of VAE to treat invasive or in situ malignancy. He et al. reported a series of 126 patients 
undergoing $\mathrm{VAB}$ and reported complete excision rates of $37.3 \%$ [36]. However, this was a retrospective series of patients undergoing VAB for radiologically benign lesions of maximum size $3 \mathrm{~cm}$, where the intent of the procedure was diagnostic rather than therapeutic. Given the findings of previous studies, which suggest that much higher rates of complete excision can be achieved with careful selection of patients based on lesion size and imaging features, it seems likely that much higher rates of excision can be obtained, comparable with the rates of complete excision seen after open breast conserving surgery. There is therefore sufficient evidence regarding the efficacy and acceptability of VAE to support its repurposing for the treatment of small, impalpable and biologically favourable screen-detected breast cancers. However, to change practice in this regard will require prospective randomised evidence from a clinical trial, and the UK SMALL trial, funded by the National Institute for Health Research Health Technology Assessment programme, seeks to generate such evidence [37•].

\section{The UK SMALL Trial}

SMALL is a prospective, randomised (2:1) phase III trial of minimally invasive VAE versus conventional open surgery in patients with small, biologically favourable screen-detected breast cancers. The study aims to generate high-quality evidence to support safely reducing the extent of surgical treatment for a highly selected group of patients. The key study questions in SMALL are whether:

- The extent of surgical treatment in this patient group can be safely reduced in the context of standard adjuvant radiotherapy and endocrine therapy

- VAE is non-inferior to conventional surgery in terms of the need for a second procedure to achieve complete resection of the tumour

- There is an acceptable rate of local recurrence following VAE with long-term follow up.

Patients randomised to VAE will undergo placement of the clip at the tumour bed at the time of their procedure. Completeness of excision will be assessed radiologically following VAE, and patients in whom no residual lesion is visible will proceed to adjuvant radiotherapy to the breast and endocrine therapy. The study schema and key eligibility criteria are shown in Fig. 1.

SMALL has a novel trial design, with two co-primary endpoints. The first is a randomised non-inferiority comparison of the requirement for a second procedure to achieve complete resection between the two treatment approaches. The requirement for re-excision following breast conserving surgery is estimated at $20 \%$, based on current UK data from the Association of Breast Surgery and Public Health England Breast Screening Audit [13]. Given the complete excision rates following VAE for lesions $<15 \mathrm{~mm}$ in diameter have been reported as approaching $100 \%$, it was considered that incomplete excision rates should be comparable in both arms [26]. Therefore, after consultation with patient advocates, it was felt that a non-inferiority margin of $10 \%$ would be appropriate for this outcome. While this is a wider noninferiority margin than might normally be considered acceptable in an oncology study, this is not a survival or recurrence outcome. Patients with failed VAE will require standard surgery, which would have been their treatment pathway had they not entered the study. Therefore, a wide margin non-inferiority was considered acceptable for this trial.

Subsequent single-arm follow-up of the VAE arm patients will then allow determination of local recurrence rates in this group. Local recurrence rates following conventional surgical excision and whole or partial breast radiotherapy are known to be extremely low (1\% at 5 years in the UK IMPORT-LOW study) [38]. SMALL aims to show that the surgical treatment of good prognosis tumours can be de-escalated while maintaining acceptable local control. With such low event rates, a randomised non-inferiority comparison of local recurrence rates in the two study arms is numerically prohibitive. However, to change practice, it is necessary to demonstrate that local recurrence rates following VAE remain low, and therefore, local recurrence rates in the study should not exceed a pre-defined limit of $3 \%$, and this will be continually monitored throughout the study by the Independent Data Monitoring Committee. Again, this threshold has been set in close conjunction with patient advocates, and is set in recognition that in this cohort, local recurrence is not a lifethreatening condition and is salvageable with further surgical treatment. This "acceptable threshold" approach is in line with other risk-adapted studies evaluating treatment de-escalation in the UK [39].

Following VAE, completeness of lesion excision will be assessed radiologically, with placement of a marker clip at the tumour bed at the time of the procedure. Post-procedure mammograms will be carried out both to check clip position and to ensure lesion removal. Where significant haematoma obscures radiological assessment, ultrasound, haematoma aspiration, and repeat mammography at a 4-6-week interval will be used as necessary to confirm lesion exision. These findings will be taken in conjunction with the operating clinician's impression of completeness of removal at the time of the initial procedure. No formal histopathological assessment of excision margins will be carried out following VAE. It is important to recognise that even after conventional surgery, a clear resection margin does not mean that there is no residual 
Fig. 1 The SMALL study schema and key eligibility criteria

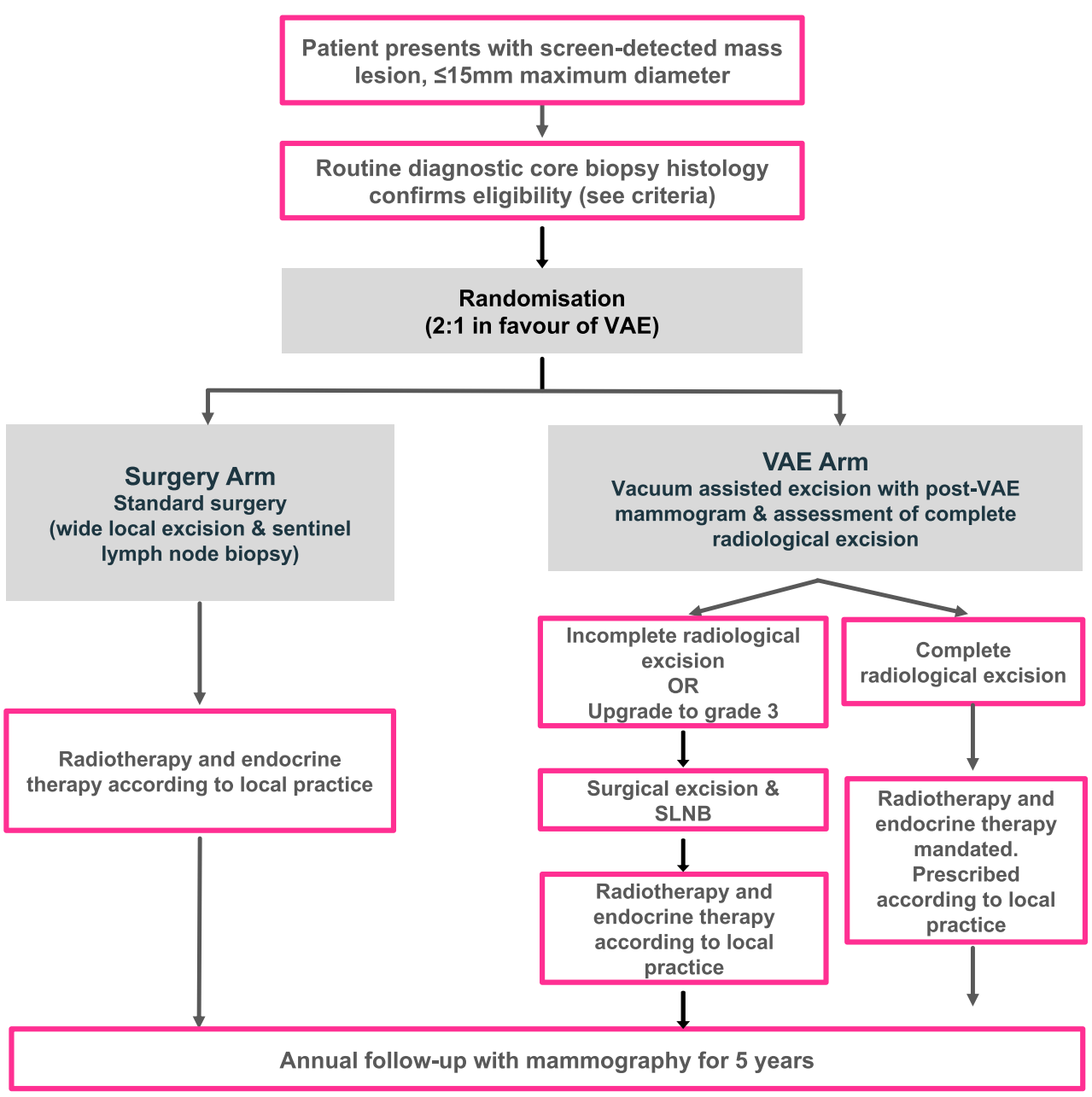

Key Eligibility Criteria

- Female aged $\geq 47$ years old with screen-detected breast cancer

- $<15 \mathrm{~mm}$ maximum tumour diameter (mammogram or ultrasound)

- No associated mammographic microcalcification

- Unifocal disease

- Grade 1 disease

- ER/PR strongly positive (Allred $\geq 7$ or equivalent)

- HER2 negative

- Negative clinical/radiological axillary staging tumour in the breast; rather, it implies that the remaining tumour burden is sufficiently low that it can be adequately treated with adjuvant therapies. Data suggests that where additional surgery is not carried out to clear focally involved margins, acceptable local recurrence rates $(<3 \%)$ can be obtained at 5 years in the context of adjuvant radiotherapy and endocrine therapy [40]. Patients in the VAE arm will have adjuvant radiotherapy and endocrine therapy mandated; this can be prescribed according to local protocols. Adjuvant radiotherapy and endocrine therapy in the standard surgery arm of the trial may be given (or omitted) according to local MDT practice, including the use of partial breast radiotherapy in line with UK guidance.

Secondary outcome measures in the trial will assess the psychological and aesthetic impact of VAE in comparison with standard surgery and a full health economic analysis will be carried out, as there are significant healthcare cost savings associated with VAE rather than standard surgery. SMALL will also employ an integrated Qualitative Recruitment Intervention $(\mathrm{QRI})$ to explore challenges and barriers to trial participation. The QRI is a novel approach using qualitative and mixed methods research which is aimed at optimising recruitment and informed consent to the study and has been 
shown to support recruitment to time and target in other challenging randomised trials $[41,42]$. SMALL will require recruitment of 800 patients over 4 years, with a subsequent follow-up period of 5 years.

\section{Conclusions}

It is likely that as our understanding of tumour biology continues to evolve, our ability to identify truly good prognosis tumours will improve, using both conventional histopathological parameters and molecular assays. In this context, it becomes ever more important to minimise the burden of treatment and long-term sequelae for women diagnosed with such cancers. Minimally invasive approaches, such as vacuum-assisted excision, appear to offer an opportunity to stratify surgical treatment according to risk and in the UK SMALL forms an integral part of a portfolio of risk-adapted breast cancer treatment trials. These include the biomarker-driven PRIMETIME trial, evaluating the omission of radiotherapy in breast cancers at very low risk of local recurrence [39]. Such trials will generate high-quality evidence to pave the way for the development of future risk-adapted strategies for breast cancer treatment, which seek to reduce treatment intensity and toxicity while maintaining optimal disease control. This will allow us to provide adequate information to our patients to enable them to make truly informed, shared decisions with their clinicians to individualise all aspects of breast cancer treatment in the twenty-first century.

\section{Declarations}

Human and Animal Rights and Informed Consent This article does not contain any studies with human or animal subjects performed by any of the authors.

Conflict of Interest Stuart McIntosh reports grants from UK National Institute for Health Research, during the conduct of the study; personal fees and other from Roche; other from ALMAC Diagnostics; personal fees and other from Daiichi-Sankyo; personal fees from BARD; and other from Novartis, outside the submitted work.

Open Access This article is licensed under a Creative Commons Attribution 4.0 International License, which permits use, sharing, adaptation, distribution and reproduction in any medium or format, as long as you give appropriate credit to the original author(s) and the source, provide a link to the Creative Commons licence, and indicate if changes were made. The images or other third party material in this article are included in the article's Creative Commons licence, unless indicated otherwise in a credit line to the material. If material is not included in the article's Creative Commons licence and your intended use is not permitted by statutory regulation or exceeds the permitted use, you will need to obtain permission directly from the copyright holder. To view a copy of this licence, visit http://creativecommons.org/licenses/by/4.0/.

\section{References}

Papers of particular interest, published recently, have been highlighted as:

- Of importance

•- Of major importance

1. Bray F, Ferlay J, Soerjomataram I, Siegel RL, Torre LA, Jemal A. Global cancer statistics 2018: GLOBOCAN estimates of incidence and mortality worldwide for 36 cancers in 185 countries. CA Cancer J Clin. 2018;68(6):394-424.

2. Bray F, McCarron P, Parkin DM. The changing global patterns of female breast cancer incidence and mortality. Breast Cancer Res. 2004;6(6):229-39.

3. Quinn M, Allen E. Changes in incidence of and mortality from breast cancer in England and Wales since introduction of screening. United Kingdom Association of Cancer Registries. BMJ. 1995;311(7017):1391-5.

4. Narod SA, Iqbal J, Miller AB. Why have breast cancer mortality rates declined? J Cancer Policy. 2015;5:8-17.

5. Welch HG, Prorok PC, O'Malley AJ, Kramer BS. Breast-Cancer Tumor Size, Overdiagnosis, and Mammography Screening Effectiveness. N Engl J Med. 2016;375(15):1438-47. Analysis of SEER data showing that screening mammography results in the additional detection of small tumours which are likely overdiagnoses and that reduced mortality is a predominantely due to improved systemic therapy.

6. Hudis CA. Biology before Anatomy in Early Breast CancerPrecisely the Point. N Engl J Med. 2015;373(21):2079-80.

7.• Lannin DR, Wang S. Are small breast cancers good because they are small or small because they are good? N Engl J Med. 2017;376(23):2286-91. Analysis of SEER dataset characterising the biological characteristics of breast tumours most likely to be overdiagnosed in mammographic screening programmes.

8. Sparano JA, Gray RJ, Makower DF, Pritchard KI, Albain KS, Hayes DF, et al. Prospective validation of a 21-gene expression assay in breast cancer. N Engl J Med. 2015;373(21):2005-14.

9. Etzioni R, Xia J, Hubbard R, Weiss NS, Gulati R (2014). A reality check for overdiagnosis estimates associated with breast cancer screening. Journal of the National Cancer Institute.106(12).

10. UK Independent Panel on Breast Cancer Screening. The benefits and harms of breast cancer screening: an independent review. Lancet. 2012;380(9855):1778-86.

11. Fisher B, Anderson S, Bryant J, Margolese RG, Deutsch M, Fisher ER, et al. Twenty-year follow-up of a randomized trial comparing total mastectomy, lumpectomy, and lumpectomy plus irradiation for the treatment of invasive breast cancer. N Engl J Med. 2002;347(16): 1233-41.

12. Veronesi U, Cascinelli N, Mariani L, Greco M, Saccozzi R, Luini A, et al. Twenty-year follow-up of a randomized study comparing breast-conserving surgery with radical mastectomy for early breast cancer. N Engl J Med. 2002;347(16):1227-32.

13. Association of Breast Surgery and Public health England. An audit of screen-detected breast cancers for the year of screening April 2018 to March 2019 2020. Available from: https:// associationofbreastsurgery.org.uk/professionals/audit/nhs-breastscreening-programme-audit/.

14. Cil TD, Cordeiro E. Complications of oncoplastic breast surgery involving soft tissue transfer versus breast-conserving surgery: an analysis of the NSQIP database. Ann Surg Oncol. 2016;23(10): 3266-71.

15. Al-Ghazal S, Fallowfield L, Blamey R. Does cosmetic outcome from treatment of primary breast cancer influence psychosocial morbidity? Eur J Surg Oncol. 1999;25(6):571-3. 
16. McCahill LE, Single RM, Aiello Bowles EJ, Feigelson HS, James TA, Barney T, et al. Variability in reexcision following breast conservation surgery. JAMA : the journal of the American Medical Association. 2012;307(5):467-75.

17. Moran M, Schnitt S, Giuliano A. al e. Society of Surgical Oncology-American Society for Radiation Oncology consensus guideline on margins for breast-conserving surgery with wholebreast irradiation in stages I and II invasive breast cancer. Ann Surg Oncol. 2014;21(3):704-16.

18. Mansel RE, Fallowfield L, Kissin M, Goyal A, Newcombe RG, Dixon JM, et al. Randomized multicenter trial of sentinel node biopsy versus standard axillary treatment in operable breast cancer: the ALMANAC Trial. J Natl Cancer Inst. 2006;98(9):599-609.

19. Giuliano AE, Ballman KV, Mc Call L, Beitsch PD, Brennan MB, Kelemen PR, et al. Effect of axillary dissection vs no axillary dissection on 10 -year overall survival among women with invasive breast cancer and sentinel node metastasis: the ACOSOG Z0011 (Alliance) randomized clinical trial. JAMA : the journal of the American Medical Association, 26. 2017;318(10):918. 10 year follow up of ACOSOG Z0011 trial, showing that completion axillary dissection in T1/2 invasive breast cancer following a positive sentinel lymph node biopsy does not impact recurrence or survival rates.

20. O'Connell RL, Rusby JE, Stamp GF, Conway A, Roche N, Barry P, et al. Long term results of treatment of breast cancer without axillary surgery - predicting a SOUND approach? European journal of surgical oncology : the journal of the European Society of Surgical Oncology and the British Association of Surgical Oncology. 2016;42(7):942-8.

21. Gentilini O, Veronesi U. Abandoning sentinel lymph node biopsy in early breast cancer? A new trial in progress at the European Institute of Oncology of Milan (SOUND: Sentinel node vs Observation after axillary UltraSouND). Breast. 2012;21(5):67881.

22. Reimer T, Stachs A, Nekljudova V, Loibl S, Hartmann S, Wolter K, et al. Restricted axillary staging in clinically and sonographically node-negative early invasive breast cancer (c/iT1-2) in the context of breast conserving therapy: first results following commencement of the Intergroup-Sentinel-Mamma (INSEMA) Trial. GebFra DGGG-Gesellschaftsausgaben. 2017;01:149-57.

23. van Roozendaal LM, Vane MLG, van Dalen T, van der Hage JA, Strobbe LJA, Boersma LJ, et al. Clinically node negative breast cancer patients undergoing breast conserving therapy, sentinel lymph node procedure versus follow-up: a Dutch randomized controlled multicentre trial (BOOG 2013-08). BMC Cancer. 2017;17(1):459.

24. Mauri G, Sconfienza LM, Pescatori LC, Fedeli MP, Ali M, Di Leo $\mathrm{G}$, et al. Technical success, technique efficacy and complications of minimally-invasive imaging-guided percutaneous ablation procedures of breast cancer: a systematic review and meta-analysis. Eur Radiol. 2017;27(8):3199-3210. Comprehensive review of minimally invasive approaches to the image-guided percutaneous, ablation of breast cancers showing technical success rates of 94$\mathbf{9 7 \%}$ and efficacy rates of $67-81 \%$ for a range of approaches.

25. Whitworth P, Schonholz S, Phillips R, Robertson Y, Ruiz A, Winchester S, et al. Minimally invasive intact excision of highrisk breast lesions and small breast cancers: the Intact Percutaneous Excision (IPEX) Registry. Ann Surg Oncol. 2019;26(4):954-60. Registry study of percutaneous exision of small in situ and invasive breast cancers, showing clear margin status in $81 \%$ of cases.

26. Sperber F, Blank A, Metser U, Flusser G, Klausner JM, LevChelouche D. Diagnosis and treatment of breast fibroadenomas by ultrasound-guided vacuum-assisted biopsy. Arch Surg. 2003;138(7):796-800.
27. Baez E, Huber A, Vetter M, Hackeloer B. Minimal invasive complete excision of benign breast tumors using a three-dimensional ultrasound-guided mammotome vacuum device. Ultrasound Obstet Gynecol. 2003;21(3):267-72.

28. Fine RE, Boyd BA, Whitworth PW, Kim JA, Harness JK, Burak WE. Percutaneous removal of benign breast masses using a vacuum-assisted hand-held device with ultrasound guidance. Am J Surg. 2002;184(4):332-6.

29. Liberman L, Menell JH. Breast imaging reporting and data system (BI-RADS). Radiologic Clinics. 2002;40(3):409-30.

30. McMahon MA, Haigh I, Chen Y, Millican-Slater RA, Sharma N. Role of vacuum assisted excision in minimising overtreatment of ductal atypias. Eur J Radiol. 2020;131:109258.

31. Bennett I, de Viana D, Law M, Saboo A. Surgeon-performed vacuum-assisted biopsy of the breast: results from a multicentre australian study. World J Surg. 2020;44(3):819-24.

32. Fine R, Israel $\mathrm{P}$, Walker L. al e. A prospective study of the removal rate of imaged breast lesions by an 11-gauge vacuum-assisted biopsy probe system. Am J Surg. 2001;182(4):335-40.

33. Perretta T, Lamacchia F, Ferrari D, Beninati E. F DIT, V DES, et al. Evaluation of ultrasound-guided 8-gauge vacuum-assisted excision system for the removal of US-detectable breast lesions. Anticancer Res. 2020;40(3):1719-29.

34. Eller A, Janka R, Lux M. al e. Stereotactic vacuum-assisted breast biopsy (VABB)-a patients' survey. Anticancer Res. 2014;34(7): 3831-7.

35. Pinder SE, Shaaban A, Deb R, Desai A, Gandhi A, Lee AHS, et al. NHS Breast Screening multidisciplinary working group guidelines for the diagnosis and management of breast lesions of uncertain malignant potential on core biopsy (B3 lesions). Clin Radiol. 2018;73(8):682-92.

36. He XF, Ye F, Wen JH, Li SJ, Huang XJ, Xiao XS, et al. High residual tumor rate for early breast cancer patients receiving vacuum-assisted breast biopsy. J Cancer. 2017;8(3):490-6.

37. Morgan J, Potter S, Sharma N, McIntosh SA. The SMALL trial: a big change for small breast cancers. Clin Oncol (R Coll Radiol). 2019;31(9):659-63. Outlines the UK randomised trial comparing VAE with standard surgery for the management of small, screen-detected breast cancers with favourable biological characteristics.

38. Coles CE, Griffin CL, Kirby AM, Titley J, Agrawal RK, Alhasso A, et al. Partial-breast radiotherapy after breast conservation surgery for patients with early breast cancer (UK IMPORT LOW trial): 5year results from a multicentre, randomised, controlled, phase 3, non-inferiority trial. Lancet. 2017;390(10099):1048-60.

39. Kirwan C, Coles C, Bliss J. on behalt of the PRIMETIME Protocol Working Group. It's PRIMETIME. Postoperative avoidance of radiotherapy: biomarker selection of women at very low risk of local recurrence. Clin Oncol. 2016;28:594-6.

40. Vos EL, Siesling S, Baaijens MHA, Verhoef C, Jager A, Voogd $\mathrm{AC}$, et al. Omitting re-excision for focally positive margins after breast-conserving surgery does not impair disease-free and overall survival. Breast Cancer Res Treat. 2017;164(1):157-67.

41. Donovan JL, Rooshenas L, Jepson M, Elliott D, Wade J, Avery K, et al. Optimising recruitment and informed consent in randomised controlled trials: the development and implementation of the Quintet Recruitment Intervention (QRI). Trials. 2016;17(1):283.

42. Rooshenas L, Scott LJ, Blazeby JM, Rogers CA, Tilling KM, Husbands S, et al. The QuinteT Recruitment Intervention supported five randomized trials to recruit to target: a mixed-methods evaluation. J Clin Epidemiol. 2019;106:108-20.

Publisher's Note Springer Nature remains neutral with regard to jurisdictional claims in published maps and institutional affiliations. 